\title{
A logical explanation of structurally unfit X-ray diffraction peaks in nanoferroelectrics
}

\author{
C M DUDHE ${ }^{1, *}$, B K SAKHARE ${ }^{2}$, S S PANCHBHAI ${ }^{3}$, S J KHAMBADKAR ${ }^{1}$, N V DHOKE ${ }^{1}$, \\ C P CHAUDHARI ${ }^{1}$ and U A PALIKUNDWAR ${ }^{4}$ \\ ${ }^{1}$ Department of Physics, Institute of Science, Nagpur 440001, India \\ ${ }^{2}$ Department of Physics, S.D.S.M. College, Palghar 401404, India \\ ${ }^{3}$ Department of Physics, Vidyabharti College, Seloo 442104, India \\ ${ }^{4}$ Department of Physics, R.T.M. Nagpur University, Nagpur 440033, India \\ *Author for correspondence (chandraguptadudhe@ gmail.com)
}

MS received 26 December 2016; accepted 5 June 2017; published online 5 February 2018

\begin{abstract}
In the present paper we suggest the cause and solution of some unidentified X-ray diffraction (XRD) peaks in ferroelectric nanoparticles. Indeed, a relationship between the structurally unfit XRD peaks and domains in the ferroelectric nanoparticles is suggested. $\mathrm{BaTiO}_{3}, \mathrm{PbTiO}_{3}$ and $\mathrm{Sr}_{0.5} \mathrm{Ba}_{0.5} \mathrm{Nb}_{2} \mathrm{O}_{6}$ nanoparticles were used as trial samples. Diffraction of $\mathrm{X}$-rays by domain grating was considered for the occurrence of unfit peaks. It was found that domain widths corresponding to some structurally unfit minor peaks of all three trail samples show good agreement to the values estimated from the transmission electron microscopy images. The study can be used to estimate the width of nanodomains (within 5-10 $\AA$ ) in ferroelectric nanoparticles. Thus, the study seems to be highly important for the advancement of nanoferroelectricity.
\end{abstract}

Keywords. X-ray diffraction; domains; ferroelectrics; nanoparticles; transmission electron microscopy.

\section{Introduction}

Inorganic ferroelectric single crystals have been extensively studied for applications like piezoelectric filters, pyroelectric detectors, imaging devices, optical memories, modulators, etc. $[1,2]$. These applications are due to their excellent dielectric non-linearity, polarization reversal, photorefractive, piezoelectric and domain dynamic properties $[1,2]$. Despite such prosperous background, as far as the contribution in nanotechnology and nanodevices is concerned, ferroelectrics seem to be lagging behind. This is probably due to the negative results of some initial investigations [3-5].

At the end of last century, Zhong et al [3], Chattopadhyay et al [4] and Sun et al [5] reported that the ferroelectric behaviour disappears when the size of the particles reduces to a nanoscale. Although at later stages such problems were resolved by means of many experimental studies [6-12], progress lagged. Another problem that creates some kinds of hurdles for researchers in the field of nanoferroelectrics is the presence of unknown diffraction peaks in powder $\mathrm{X}$-ray diffraction (XRD) pattern. We also experienced such problems in $\mathrm{BaTiO}_{3}$ and $\mathrm{PbTiO}_{3}$ nanoparticles [9,11]. Up to the limited value of relative intensity, such peaks can be ignored just by stating 'insignificant minor peaks due to weak unknown phase or impurity'. However, the presence of such peaks induces some kinds of uncertainty about the synthesis technique and/or structure of nanoparticles. This may hamper the progress of research in the field of nanoferroelectrics.
Earlier in 1948, Kay [13] observed the effect of twinning on X-ray pattern. He found that due to twinning, the single spots on an X-ray photograph are replaced by pairs or a more complex group. It was also noted in the literature that the microstructure affects the intensity of reflection [14]. Later in 2000, Ingle and Patil [15] suggested that the presence of twin planes is a possible cause of unknown peaks in XRD pattern of ferroelectric material. These evidences indicate that there is a definite effect of twinning and hence domain structures on XRD pattern of the ferroelectrics since twinning process leads to formation of domains in the crystals.

Unfortunately, the matter remained unnoticed and no further correlation on this part of the research is reported. This is mainly because of dimension of domains. In single crystals, generally the dimension of domains is on the order of a micrometre, which is too large as compared with the wavelength of X-rays. Hence, every part of polydomain crystal under the field of view of X-ray beam seems to be a single domain and hence observations of said kind are infrequent. However, in nanoparticles, as the existence of nanodomains with the dimension comparable to the $\mathrm{X}$-ray wavelength is possible, the effect will be frequent if they arrange themselves in a grating-like form. Thus, the interaction between the domains and X-ray can be distinguished.

In the present work, we conducted the titled study on some ferroelectric nanoparticles and a relation between domain structure and unknown XRD peaks was established. The study provides the information of whether small domains on the 
order of $10 \AA$ are present or not. If they are present, the width of domains can also be estimated. This knowledge of domain and domain walls may support design high-density non-volatile information storage and ferroelectric mass storage devices based on scanning probes [16-18].

\section{Material and methods}

Trial ferroelectric compounds used for the study are prototype $\mathrm{BaTiO}_{3}$ and $\mathrm{PbTiO}_{3}$, and 'relaxor'-type $\mathrm{Sr}_{0.5} \mathrm{Ba}_{0.5} \mathrm{Nb}_{2} \mathrm{O}_{6}$. Synthesis techniques, XRD characterization, morphology of the nanoparticles and nanodomain structures of these compounds have been already studied by us, recently $[9,11,12]$. For the present study, XRD patterns of all three compounds obtained using an X-ray diffractometer (D8 Advance, Bruker, Germany) at wavelength $1.54 \AA$ in the range of $2 \theta=$ $20-50^{\circ}$ were used. The indexing of peaks was done by the 'least squares fit' method. Structurally unfit peaks were also identified. The widths of domains plus domain wall $(a+b)$ corresponding to these unfit peaks were determined using equation (2) as discussed in the next section. Spectroscopic characterization of the compounds was performed using a Fourier transform infrared spectrometer FTIR-8400S (Shimadzu). Energy-dispersive X-ray (EDX) spectrometers attached to a scanning electron microscope (Zeiss, EVO 18) and a transmission electron microscope (TEM, TECNAI G2 20 Ultratwin, FEL, Netherlands) were used to identify the constituent elements of the compounds.

Selected high-resolution TEM images of $\mathrm{BaTiO}_{3}$ and $\mathrm{PbTiO}_{3}$ obtained using the TEM TECNAI G2 20 Ultratwin, FEL, Netherlands, and of $\mathrm{Sr}_{0.5} \mathrm{Ba}_{0.5} \mathrm{Nb}_{2} \mathrm{O}_{6}$ using a TEM CM200, PHILIPS, were also used for the study. The selection of TEM images for the study was based on the criterion of presence of nanodomain structures in nanoparticles.

\section{Result and discussion}

\subsection{Theoretical aspect}

In 1913, while deriving the famous equation, Bragg pointed out that lattice planes in a crystal behave as lines in a diffraction grating to X-rays, i.e., crystals can be considered as space gratings [19]. The incident X-rays are reflected from lattice planes and the width of the planes (interplanar spacing) can be measured as a function of first-order diffraction angle given by the expression

$$
\theta=\sin ^{-1}(\lambda / 2 d)
$$

where $\lambda$ is the wavelength of incident X-rays and $d$ is the interplanar spacing.

In ferroelectric material, domain formation is a very common process. Domains are the regions in which electric dipoles orient differently from region to region. If the dipoles in a region make $90^{\circ}$ orientation with the direction of polarization $\left(P_{\mathrm{s}}\right)$ of the crystal as a whole, the region is called $90^{\circ}$ domain. If they make $180^{\circ}$ orientation then the region is called antiparallel domain, and so on. These domains are separated by a boundary called domain wall. This is the region where there are no electric dipole charges [20]. If such domains in a crystal are arranged periodically, they can form a domain grating. This grating may have a different behaviour to X-rays than the space grating. Therefore, X-rays diffracted from the domain grating can produce its own diffraction pattern probably dissimilar to that from the space grating. The maxima position of such pattern can be obtained by the grating equation

$$
(a+b) \sin \theta=n \lambda,
$$

where $(a+b)$ is the grating element with $a$ as slit and $b$ as opaque, $\theta$ is the angle of diffraction, $n$ is the order of diffraction, which is taken as 1 in the present case, and $\lambda$ is the wavelength of incident beam $(\sim 1.54 \AA)$.

In general, scattering of X-rays takes place from the different atoms in the cell structure, giving rise to the XRD pattern. However, in case of ferroelectric domain grating, the X-ray scattering may take place from the charged dipoles. Since there is no charge on the ferroelectric domain wall, the beam of X-ray when incident on the region of wall does not show any diffraction. On the other hand, the domain region consisting of charged dipoles (like $\mathrm{Ti}-\mathrm{O}$ or $\mathrm{Nb}-\mathrm{O}$ ) caused by the distortion in $\mathrm{Ti}-\mathrm{O}_{6}$ or $\mathrm{Nb}-\mathrm{O}_{6}$ octahedra can show diffraction of X-rays. Since such sites in crystals are very few, the intensity counts are expected to be small.

Because of absence of deviation of X-rays from the domain wall, we assumed the width of the 'domain wall' as $b$. The width of the 'domain' of course is treated as $a$.

As we assume that the XRD due to domain grating is governed by equation (2), there is a certain limitation that is mostly associated with the width of the domain grating. This concept is therefore strongly recommended for the nanodomains pattern having $2 \theta$ value that falls between 10 and $60^{\circ}$ corresponding to $(a+b)$. For example, if we take $(a+b)=5 \mathrm{~nm}$, the value of $2 \theta$ nearly equals $3.5^{\circ}$. On the other hand, if we take $(a+b)=0.2 \mathrm{~nm}$, the $2 \theta$ nearly equals $100^{\circ}$. This shows that the value of $2 \theta$ limits the $(a+b)$ values; the lower $(a+b)$ creates ambiguity with the spacing of lattice fringes, whereas the higher $(a+b)$ cannot be observed on the general scale of $2 \theta$. Our calculation shows that values are best suited when $(a+b)$ is around 5-10 $\AA$, corresponding to which the $2 \theta$ scale is approximately $20-50^{\circ}$.

\subsection{Experimental verification}

Figure 1a-c shows as-recorded XRD patterns of $\mathrm{BaTiO}_{3}$, $\mathrm{PbTiO}_{3}$ and $\mathrm{Sr}_{0.5} \mathrm{Ba}_{0.5} \mathrm{Nb}_{2} \mathrm{O}_{6}$ nanoparticles along with respective JCPDS files. We have already reported the lattice parameters of $\mathrm{BaTiO}_{3}$ as $a=b=3.995 \AA$ and $c=4.026 \AA$, 

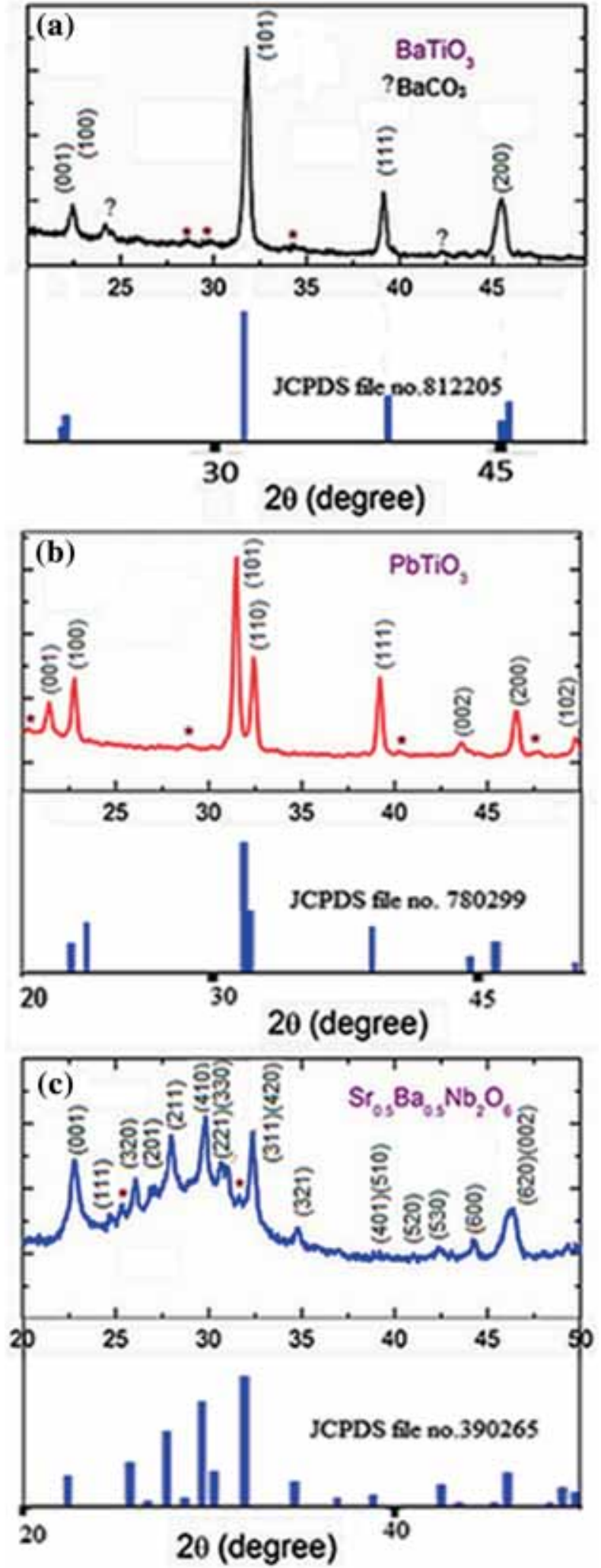

Figure 1. $\mathrm{XRD}$ patterns of (a) $\mathrm{BaTiO}_{3}$, (b) $\mathrm{PbTiO}_{3}$ and (c) $\mathrm{Sr}_{0.5} \mathrm{Ba}_{0.5} \mathrm{Nb}_{2} \mathrm{O}_{6}$ nanoparticles showing structural peaks pattern along with structurally unfit minor peaks (unfit peaks are shown by symbols '*').

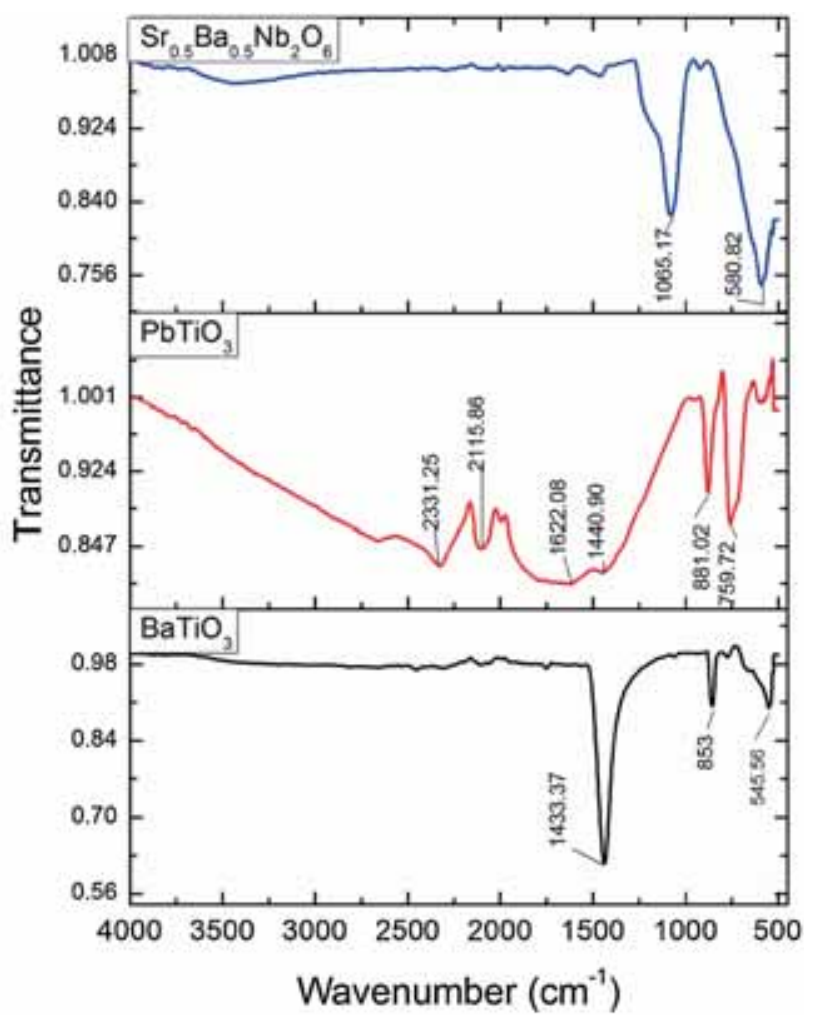

Figure 2. FTIR spectra of understudied $\mathrm{BaTiO}_{3}, \mathrm{PbTiO}_{3}$ and $\mathrm{Sr}_{0.5} \mathrm{Ba}_{0.5} \mathrm{Nb}_{2} \mathrm{O}_{6}$ nanoparticles.

and of $\mathrm{PbTiO}_{3}$ as $a=b=3.900 \AA$ and $c=4.145 \AA$ [9,11]. The lattice parameters of $\mathrm{Sr}_{0.5} \mathrm{Ba}_{0.5} \mathrm{Nb}_{2} \mathrm{O}_{6}$ nanoparticles are found to be $a=b=12.349 \AA$ and $c=3.907 \AA$, which are slightly smaller than our previous result $(a=b=$ $12.485 \AA$ and $c=3.950 \AA$ ) [12]. This may be because of preparation conditions. In the present study, the calcination temperature and duration for the synthesis of $\mathrm{Sr}_{0.5} \mathrm{Ba}_{0.5} \mathrm{Nb}_{2} \mathrm{O}_{6}$ nanoparticles were $800^{\circ} \mathrm{C}$ and $6 \mathrm{~h}$, respectively, whereas in our previous study, the respective parameters were $750^{\circ} \mathrm{C}$ and $10 \mathrm{~h}$ [12]. All these studied compounds bear tetragonal phases, which are acentric in nature and also possess domain structures; therefore, they are ferroelectric in nature. Ferroelectricity in these compounds was studied earlier by us $[9,11,12]$.

For the sake of structural verification, FTIR spectra of these compounds are shown in figure 2. It is known that vibration bands above $1000 \mathrm{~cm}^{-1}$ are mainly related to organic gases that are adsorbed on the surface of the compounds during their synthesis; therefore, from the structural point of view, such vibrations are not important. Vibrations in the fingerprint region $\left(1000-400 \mathrm{~cm}^{-1}\right)$ are related to the inorganic structures. It is found from figure 2 that the characteristic vibration bands for $\mathrm{BaTiO}_{3}$ are observed at 853 and $545.6 \mathrm{~cm}^{-1}$ and those for $\mathrm{PbTiO}_{3}$ at 881.02 and $759.72 \mathrm{~cm}^{-1}$. These vibrations can be assigned to stretching and bending vibrations of TiO-Ti. Similarly, the vibration at $580.82 \mathrm{~cm}^{-1}$ for the case of 


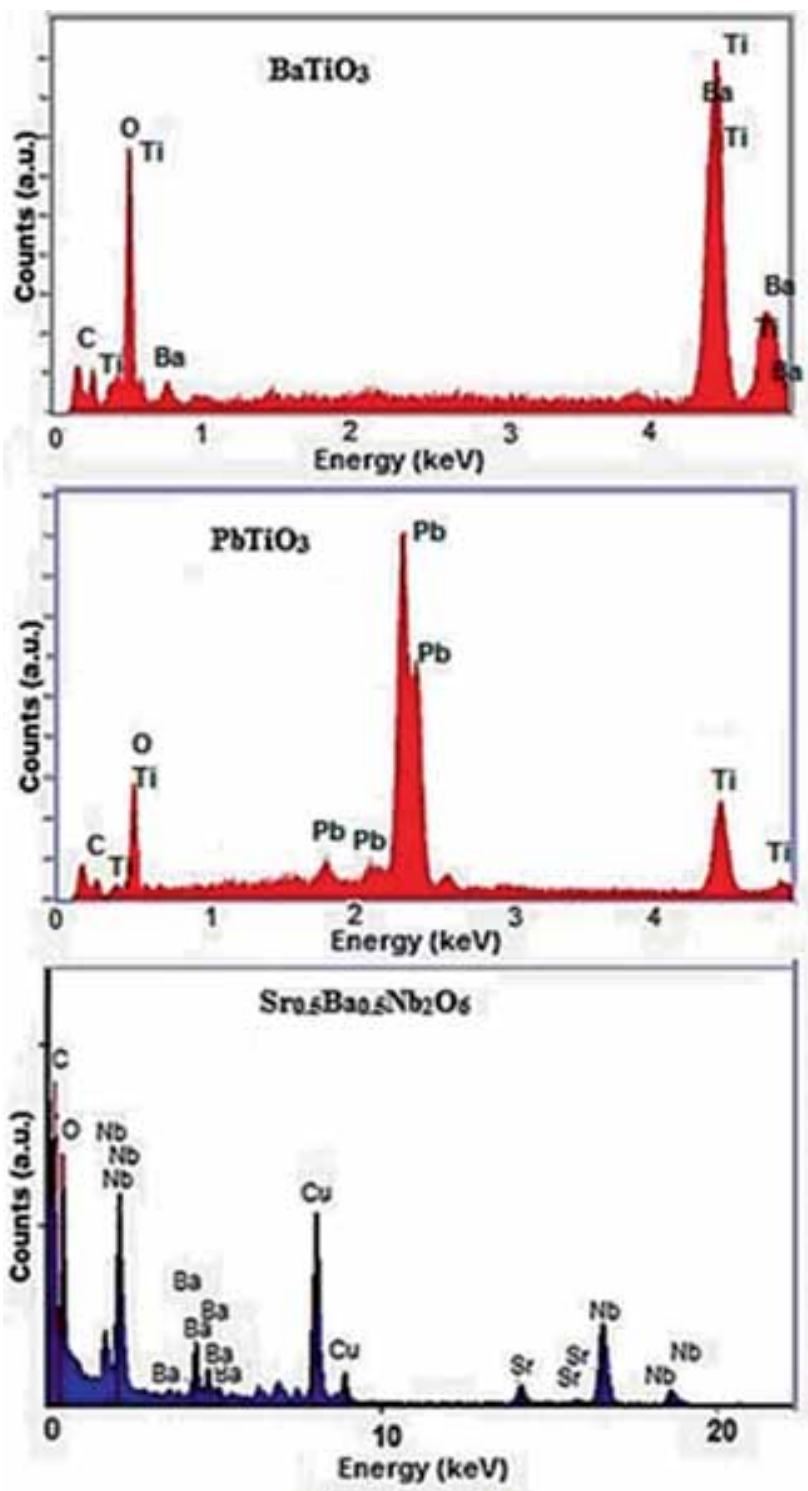

Figure 3. EDX spectra of the samples confirming constituents of the compounds.

$\mathrm{Sr}_{0.5} \mathrm{Ba}_{0.5} \mathrm{Nb}_{2} \mathrm{O}_{6}$ can be assigned to the $\mathrm{Nb}-\mathrm{O}-\mathrm{Nb}$ stretching vibration. These spectra in the fingerprint region show good agreement with the reported spectra [21-23], giving the validation of the synthesized compounds.

EDX spectra of these compounds are shown in figure 3. This figure shows the absence of foreign impurities. Note that the EDX spectrum of $\mathrm{Sr}_{0.5} \mathrm{Ba}_{0.5} \mathrm{Nb}_{2} \mathrm{O}_{6}$ obtained by the EDX spectrometer attached to the TEM spectrometer shows signals of $\mathrm{C}$ and $\mathrm{Cu}$. These signals are due to the carbon-coated copper grid. The result therefore shows that the unidentified peaks in XRD spectra (figure $1 \mathrm{a}-\mathrm{c}$ ) are due to the internal structures, like nanodomains.

In figure 1a-c, unidentified peaks are marked by symbol ‘*'. Also, two '?'-marked peaks are seen in figure 1a, which are probably due to the trace of $\mathrm{BaCO}_{3}$ (JCPDS file number 410373). Relative intensities and/or $2 \theta$ positions of $*$-marked

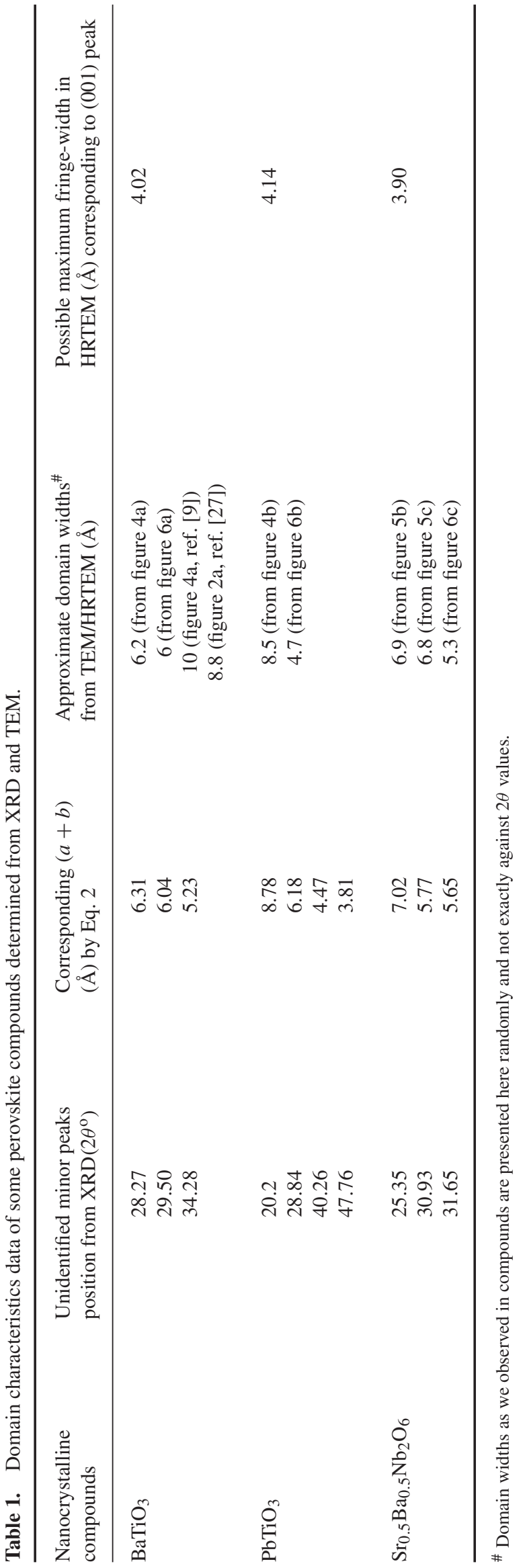



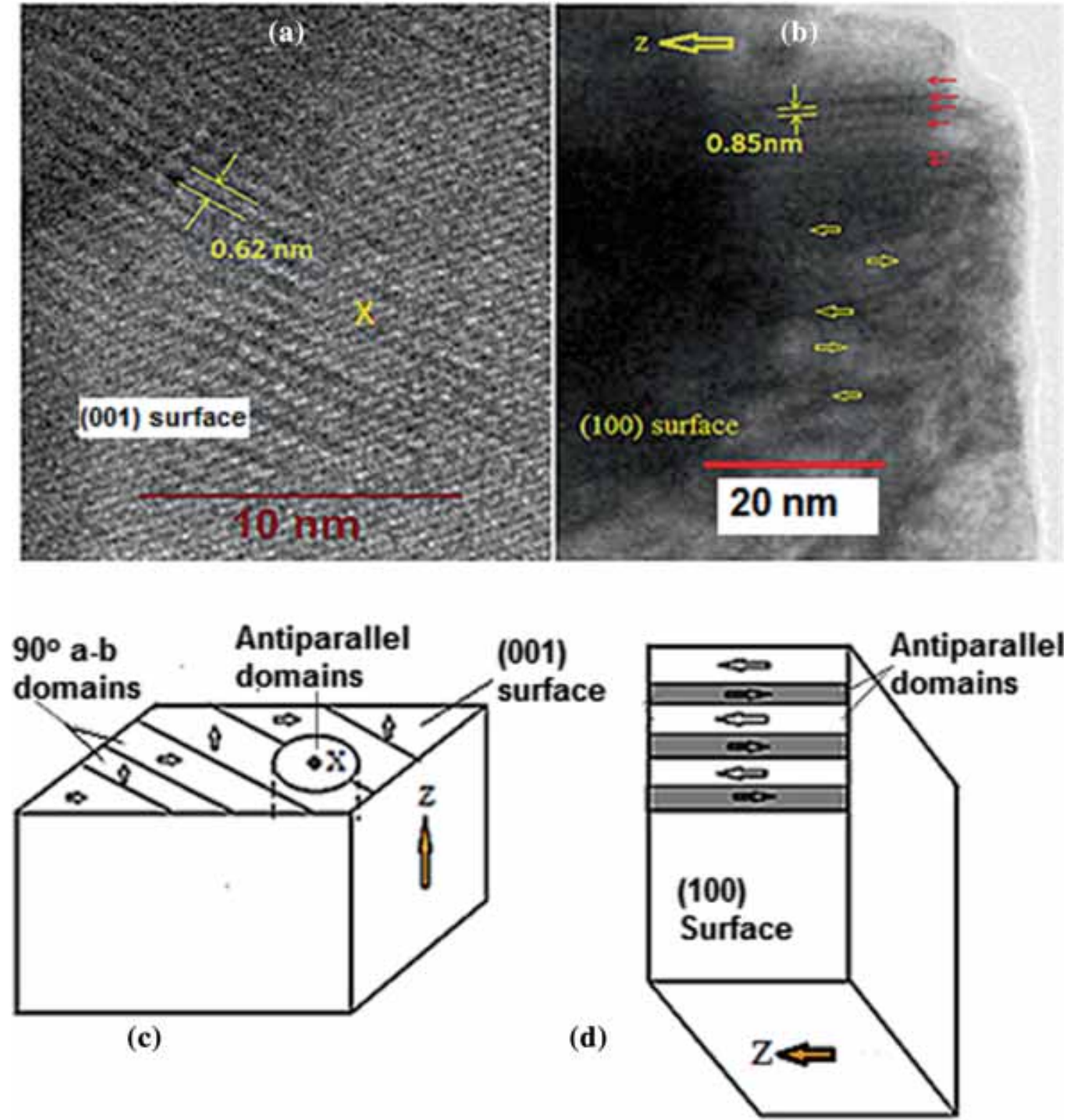

Figure 4. High-resolution TEM images of (a) $\mathrm{BaTiO}_{3}$ and (b) $\mathrm{PbTiO}_{3}$. A careful observation shows $180^{\circ}$ domain region that appears as an elliptical shallow pit surrounding the ' $\mathrm{X}$ ' in $\mathbf{a}$. Here, the direction of $P_{\mathrm{S}}$ is perpendicular to the plane of paper; $90^{\circ}$ domains run along ( $\left.1 \quad \overline{1} 0\right)$ direction. In $\mathbf{b}$, the direction of $P_{\mathrm{S}}$ is shown by a bigger arrow and red coloured arrows indicate the crowded $180^{\circ}$ domains. Domain structures in images $\mathbf{a}$ and $\mathbf{b}$ are shown schematically in c and $\mathbf{d}$, respectively. $Z$-direction indicates the direction of $P_{\mathrm{s}}$ of the crystallite as a whole.

peaks in figure 1a do not match with the JCPDS peaks file (file number 410373) of $\mathrm{BaCO}_{3}$. This indicates that the undetermined peaks (marked by $*$ ) in figure 1a are probably not related to $\mathrm{BaCO}_{3}$ trace.

It is to be noted that the compound $\mathrm{BaCO}_{3}$ has no ferroelectric properties. Since domain properties in this study are related to individual nanoparticles, the presence of trace of $\mathrm{BaCO}_{3}$ will not affect the domain pattern of the ferroelectric $\mathrm{BaTiO}_{3}$ nanoparticles. Fortunately, the ambiguity that appears in case of $\mathrm{BaTiO}_{3}$ is not found in cases of $\mathrm{PbTiO}_{3}$ and $\mathrm{Sr}_{0.5} \mathrm{Ba}_{0.5} \mathrm{Nb}_{2} \mathrm{O}_{6}$.
The $2 \theta$ positions of unidentified peaks marked in figure 1a-c, calculated domain width, including domain wall $(a+b)$ and maximum possible fringe width (i.e., $d_{h k l}$ corresponding to the first structural peak) that may appear in high-resolution transmission electron microscopy (HRTEM) of the studied compounds are given in table 1 . To compare them to the observed data, domains in these compounds were investigated.

Figure $4 \mathrm{a}$ and $\mathrm{b}$ shows the HRTEM images of studied nanoparticles of $\mathrm{BaTiO}_{3}$ and $\mathrm{PbTiO}_{3}$, respectively. Although (because of high magnification) the contrast in figure 4 is not 


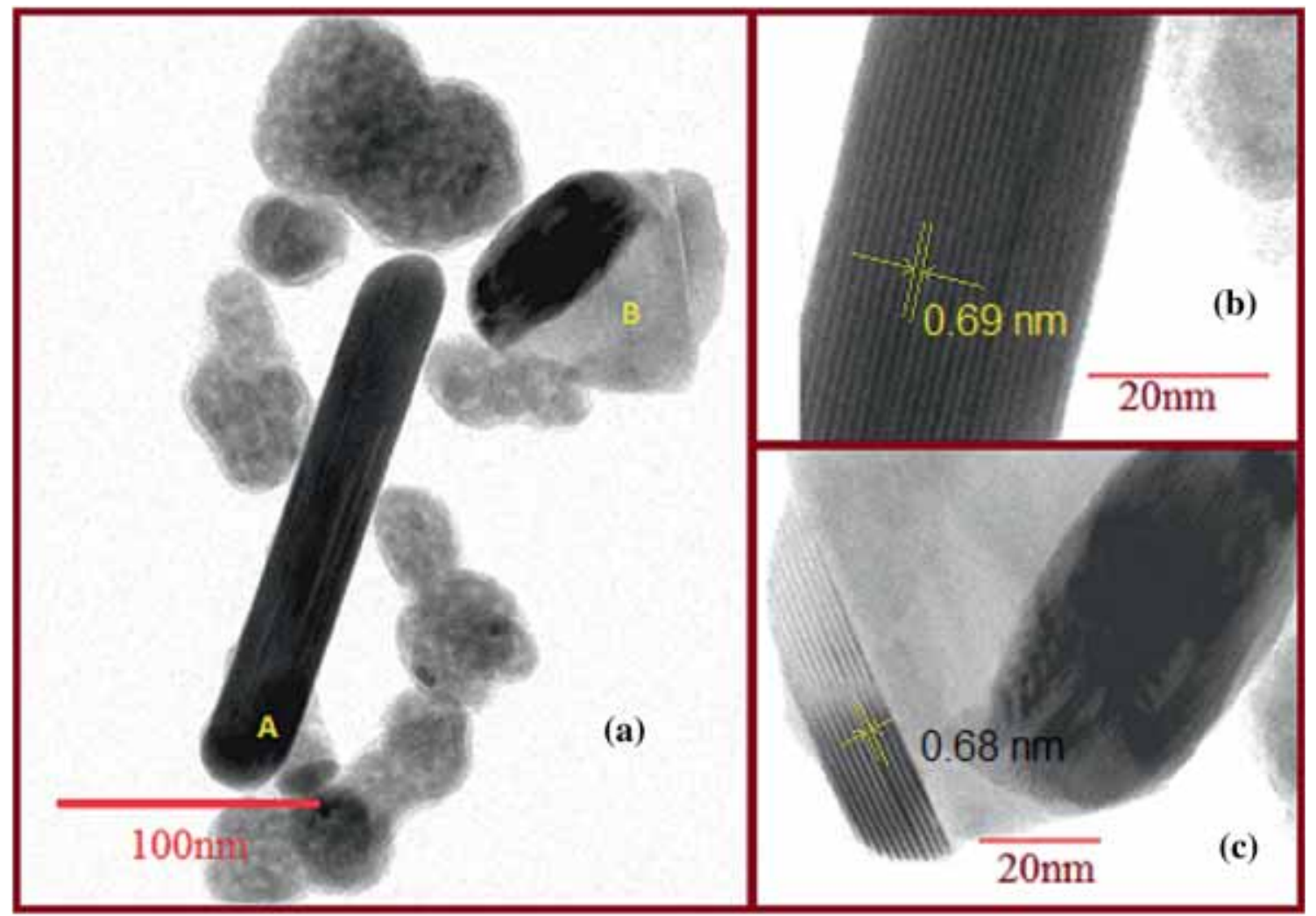

Figure 5. (a) TEM image of $\mathrm{Sr}_{0.5} \mathrm{Ba}_{0.5} \mathrm{Nb}_{2} \mathrm{O}_{6}$ nanoparticles prepared at calcined temperature $800^{\circ} \mathrm{C}$ showing typical crystallites 'A' and 'B'. (b and c) High-resolution TEM images of particles A and B, respectively; $180^{\circ}$ domains are clearly seen.

high, it is quite sufficient to understand the domain structures. Figure 4 a shows some interesting features. A careful observation shows that there is a $180^{\circ}$ domain that appears as a shallow pit. This site is marked as X. The direction of polarization $P_{\mathrm{s}}$ of this domain is, for example, $-Z$ axis. Since orientation of the $P_{\mathrm{s}}$ of the crystal as a whole in tetragonal phase tends to align along $Z$-direction, the field of view seems to be a pseudocubic (001) surface. On this surface, beside approximately (110) directional weak lattice fringes, some (1ī0) directional laminar regions are observed. The width of each such bright region is about $6.2 \AA$, which is sufficiently larger than the maximum possible fringe width (4.02 ̊̊), indicating that (1ī0) directional laminar regions are the nanodomains. Their linear arrangement on pseudocubic (001) surface shows that they are $a-b 90^{\circ}$ domains $[9,20,24,25]$. A clear picture of domains in figure $4 \mathrm{a}$ can be understood from the schematic arrangement given in figure $4 \mathrm{c}$.

The situation in figure $4 \mathrm{~b}$ is different from that in figure 4a. It shows dark and moderately bright islands, which are extended along approximately horizontal direction of the observed plane. Similar kinds of situation are already discussed in our previous paper [11], according to which these islands are antiparallel or $180^{\circ}$ domains with directions of polarization $P_{\mathrm{s}}$ along $Z$-axis, as marked by arrows. The area under the field of view is a pseudocubic (100) surface. A bigger arrow shows the direction of polar axis
$(Z)$. Hence, the crowded domains seen at the upper part in figure $4 \mathrm{~b}$ seem to be $180^{\circ}$ or antiparallel domains. This can also be understood from the schematic geometry given in figure $4 \mathrm{~d}$. In figure $4 \mathrm{~b}$, the width of domains was found be $\sim 8.5 \AA$.

The domain situation in $\mathrm{Sr}_{0.5} \mathrm{Ba}_{0.5} \mathrm{Nb}_{2} \mathrm{O}_{6}$ compound is relatively simple as only antiparallel domains similar to those in figure $4 \mathrm{~d}$ exist in tetragonal tungsten bronze (TTB)-like structures $[25,26]$. Figure 5a, the TEM image of studied $\mathrm{Sr}_{0.5} \mathrm{Ba}_{0.5} \mathrm{Nb}_{2} \mathrm{O}_{6}$ nanoparticles, shows rod-like (particle $\mathrm{A}$ ) and irregular (particle $\mathrm{B}$ and other) morphologies. Figure $5 b$ and $c$ shows the HRTEM images of particles A and $\mathrm{B}$, respectively. These figures (figure $5 \mathrm{~b}$ and $\mathrm{c}$ ) show many dark and bright strips, with the widths of bright strips of about 6.9 and $6.8 \AA$, respectively. Here also, these widths are higher than the possible maximum fringe width (3.9 $\AA$ ), similar to the $\mathrm{BaTiO}_{3}$ and $\mathrm{PbTiO}_{3}$ cases. Hence, dark and bright strips in figure $5 \mathrm{~b}$ and $\mathrm{c}$ are antiparallel domains.

Thus, by comparing the observed domain widths and $(a+b)$ values as given in table 1 , it is clear that the closefluxed $90^{\circ}$ domain of $\mathrm{BaTiO}_{3}$ as seen in figure 4 a produces the grating diffraction pattern corresponding to which the maximum appears at $2 \theta=28.27^{\circ}$; further, $180^{\circ}$ domain patterns of $\mathrm{PbTiO}_{3}$ and $\mathrm{Sr}_{0.5} \mathrm{Ba}_{0.5} \mathrm{Nb}_{2} \mathrm{O}_{6}$ as observed in figures $4 \mathrm{~b}$ and $5 \mathrm{~b}$ and c produce similar effects at $2 \theta=20.2$ and $25.35^{\circ}$, 


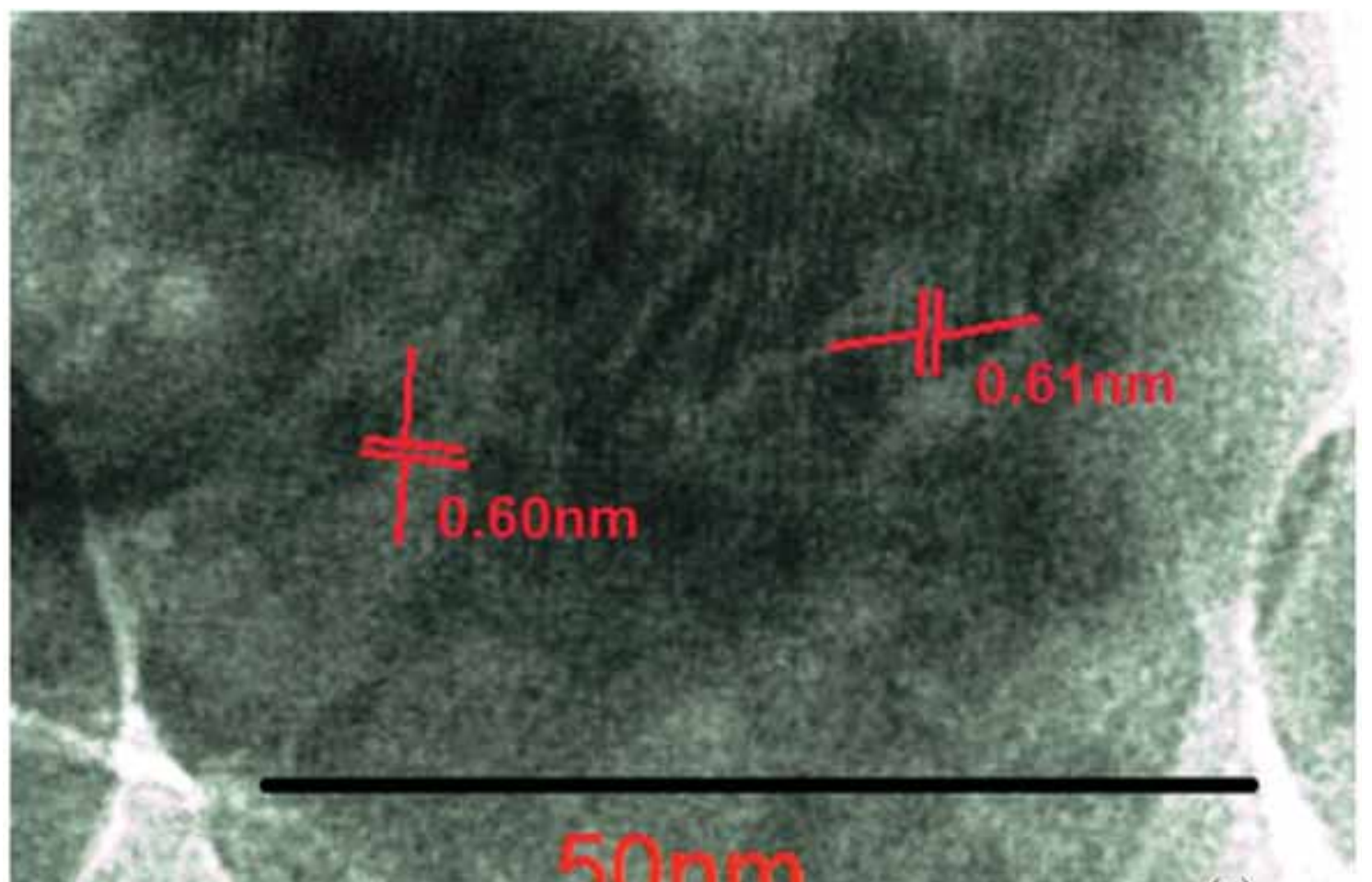

(a)
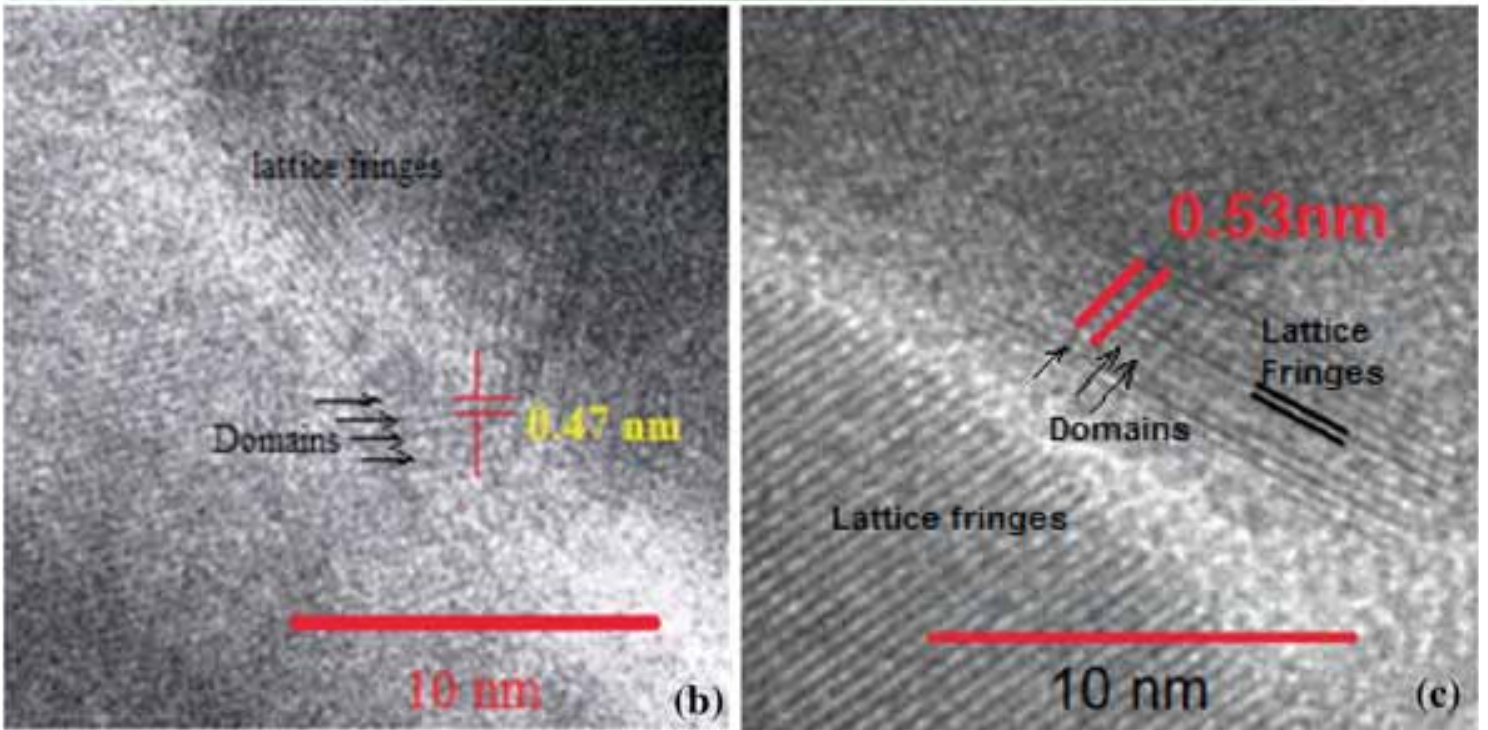

Figure 6. Another set of TEM/HRTEM images of (a) $\mathrm{BaTiO}_{3}$, (b) $\mathrm{PbTiO}_{3}$ and (c) $\mathrm{Sr}_{0.5} \mathrm{Ba}_{0.5} \mathrm{Nb}_{2} \mathrm{O}_{6}$ nanoparticles.

respectively. In fact, table 1 shows slight discrepancies in $(a+b)$ and domain widths for the afore-mentioned cases. This is expected as $(a+b)$ includes a negligibly small domain-wall width.

To concretize the relationship, another set of TEM/HRTEM images comprising domains is shown in figure 6. Domains and lattice fringes are clearly differentiated in figure $6 \mathrm{~b}$ and $\mathrm{c}$. The width of domains in $\mathrm{BaTiO}_{3}$,
$\mathrm{PbTiO}_{3}$ and $\mathrm{Sr}_{0.5} \mathrm{Ba}_{0.5} \mathrm{Nb}_{2} \mathrm{O}_{6}$ are estimated from figure $6 \mathrm{a}$, $\mathrm{b}$ and $\mathrm{c}$, respectively, and depicted in table 1 . Also, values of domain widths of studied compounds were re-determined from the TEM images of compounds reported previously by us $[9,27]$. These values are also given in table 1 with the reference of figure and published paper. These measured values of domain width show good agreements to the values of $(a+b)$ as determined from equation (2). 


\section{Conclusion}

Structurally unfit XRD peaks of $\mathrm{BaTiO}_{3}, \mathrm{PbTiO}_{3}$ and $\mathrm{Sr}_{0.5}$ $\mathrm{Ba}_{0.5} \mathrm{Nb}_{2} \mathrm{O}_{6}$ nanoparticles were identified. Also, crowded domain structures were examined. It was found that the domain widths of these crowded domains show good agreements with certain values calculated from XRD patterns using the grating equation. This shows that there may be one-toone correspondence between the structurally unfit XRD peak and domain pattern. The study however is limited to nanodomains of width within 5-10 A. The study therefore seems to be highly important for the advancement of nanoferroelectricity as it may remove the uncertainty in XRD patterns to some extent. Indeed, similar studies are required for further validation.

\section{Acknowledgements}

CMD thanks UGC of India for financial support under the MRP scheme vide letter number 47-1089/14 (WRO).

\section{References}

[1] Lines M E and Glass A M 1977 Principles and applications of ferroelectrics and related materials (Oxford: Clarendon)

[2] Xu Y 1991 Ferroelectric materials and their applications (Amsterdam: North Holland)

[3] Zhong W L, Wang Y G, Zhang P L and Qu B D 1994 Phys. Rev. B $\mathbf{5 0} 698$

[4] Chattopadhyay S, Ayyub P, Palkar V R and Multani M 1995 Phys. Rev. B $\mathbf{5 2} 13177$

[5] Sun L, Chen Y F, Ma W H, Wang L W, Yu T, Zhang M S et al 1996 Appl. Phys. Lett. 683728
[6] Cho Y, Hashimoto S, Odagawa N, Tanaka K and Hiranaga Y 2006 Nanotechnology 17 S137

[7] Akdogan E K, Rawn C J, Porter W D, Payzant E A and Safari A 2005 J. Appl. Phys. 97084305

[8] McGilly L J and Gregg J M 2011 Nano Lett. 114490

[9] Dudhe C M, Nagdeote S B, Khambadkar S J, Arjunwadkar P R and Patil R R 2014 Ferroelectrics 471148

[10] Kundu T K, Jana A and Barik P 2008 Bull. Mater. Sci. 31501

[11] Dudhe C M and Khambadkar S J 2015 J. Alloys Compd. 648 92

[12] Dudhe C M, Nagdeote S B and Chaudhari C P 2015 Ferroelectrics $\mathbf{4 8 2} 104$

[13] Kay H F 1948 Acta Crystallogr. 1229

[14] Peiser H S, Rooksby H P and Wilson A J C (eds) 1955 X-ray diffraction by polycrystalline materials (London: Institute of Physics)

[15] Ingle S G and Patil N M 2000 Jpn. J. Appl. Phys. 392670

[16] Rubio-Marcos F, Campo A D, Marchet P and Fernandez J F 2015 Nat. Commun. 66594

[17] Auciello O, Scott J F and Ramesh R 1998 Phys. Today 5122

[18] Tybell T, Paruch P, Giamarchi T and Triscone J M 2002 Phys. Rev. Lett. 89097601

[19] Saxena A K 2006 Solid state physics: an introduction to solid state electronic devices (Delhi: Macmillan)

[20] Jona F and Shirane G 1962 Ferroelectric crystals (Oxford: Pergamon Press)

[21] Trivedi M K, Nayak G, Patil S, Tallapragada R M, Latiyal O and Jana S 2015 Ind. Eng. Manage. 41000166

[22] Lu X, Zhang D, Zhao Q, Wang C, Zhang W and Wei Y 2006 Macromol. Rapid Commun. 2776

[23] Mathad S N and Puri V 2012 Arch. Phys. Res. 3106

[24] Potnis P R, Tsou N T and Huber J E 2011 Materials 4417

[25] Taganstev A K, Cross L E and Fousek J 2010 Domains in ferroic crystals and thin films (New York: Springer-verlag)

[26] Jamieson P B, Abrahams S C and Brenstein J L 1968 J. Chem. Phys. 485048

[27] Dudhe C M, Khambadkar S J and Nagdeote S B 2015 Mater. Lett. 161514 